\title{
Designing a Fibrotic Microenvironment to Investigate Changes in Human Liver Sinusoidal Endothelial Cell Function
}

\author{
Andrew J. Ford ${ }^{1}$, Gaurav Jain ${ }^{2}$ and Padmavathy Rajagopalan ${ }^{1,2,3, *}$
}

1. Department of Chemical Engineering, Virginia Tech, Blacksburg VA 24061

2. School of Biomedical Engineering and Sciences, Virginia Tech, Blacksburg VA 24061

3. ICTAS Center for Systems Biology of Engineered Tissues, Virginia Tech, Blacksburg VA 24061 


\section{Author Information}

Mr. Andrew J. Ford

Department of Chemical Engineering

Virginia Tech, Blacksburg VA 24060

Email: ford89@vt.edu

Tel: 540-231-4851

Dr. Gaurav Jain

School of Biomedical Engineering and Sciences

Virginia Tech

Email: jaing@vt.edu

Tel: 540-231-4851

\section{${ }^{*}$ Corresponding Author Information:}

Prof. Padmavathy Rajagopalan

Department of Chemical Engineering

School of Biomedical Engineering and Sciences,

ICTAS Center for Systems Biology of Engineered Tissues

Virginia Tech, Blacksburg VA 24061

Email: padmar@vt.edu, Tel: 540-231-4851, 


\section{ABSTRACT}

The deposition of extracellular matrix (ECM) proteins by hepatic cells during fibrosis leads to the stiffening of the organ and perturbed cellular functions. Changes in the elasticity of liver tissue are manifested by altered phenotype in hepatic cells. We have investigated changes in human liver sinusoidal endothelial cells (hLSECs) that occur as the elastic modulus of their matrix transitions from healthy $(6 \mathrm{kPa})$ to fibrotic $(36 \mathrm{kPa})$ conditions. We have also investigated the role played by Kupffer cells in the dedifferentiation of hLSECs. We report the complete loss of fenestrae and the expression of CD31 at the surface as a result of increasing elastic moduli. LSECs exhibited a greater number of actin stress fibers and vinculin focal adhesion on the stiffer substrate, as well. A novel finding is that these identical trends can be obtained on soft $(6 \mathrm{kPa})$ substrates by introducing an inflamed microenvironment through the addition of Kupffer cells. hLSEC monocultures on $6 \mathrm{kPa}$ gels exhibited fenestrae that were $140.7 \pm$ $52.6 \mathrm{~nm}$ in diameter as well as a lack of surface CD31 expression. Co-culturing hLSECs with rat Kupffer cells (rKCs) on $6 \mathrm{kPa}$ substrates, resulted in the complete loss of fenestrae, an increase in CD31 expression and in a well-organized cytoskeleton. These results demonstrate that the increasing stiffness of liver matrices does not solely result in changes in hLSEC phenotype. Even on soft substrates, culturing hLSECs in an inflamed microenvironment can result in their dedifferentiation. Our findings demonstrate the interplay between matrix elasticity and inflammation in the progression of hepatic fibrosis.

KEY WORDS: Liver sinusoidal endothelial cells; Kupffer cells; fibrosis; fenestrae; matrix stiffness 


\section{INTRODUCTION}

Liver fibrosis is a leading cause of death [1-3]. This health condition can lead to hepatic carcinomas, renal failure, toxin-induced comas, bleeding, and a host of metabolic disorders [1]. Hepatic fibrosis distorts liver architecture causing resistance to blood flow resulting in organ dysfunction [1-3]. At its core, hepatic fibrosis is an uncontrolled wound healing mechanism. Alcohol abuse, obesity, diabetes or hepatitis $C$ viral infections are some initiating events that induce fibrosis [2]. When hepatic cells experience altered signaling they respond by secreting inflammatory molecules and extra-cellular matrix (ECM) proteins $[1,4,5]$. These secretions change the rigidity and chemical properties of liver matrices that cells are exposed to, thereby resulting in altered cellular phenotypes [1]. As hepatic fibrosis occurs, an abundance of ECM proteins are produced by hepatic stellate cells (HSCs) and accumulate in the region between the sinusoidal endothelium and the liver parenchyma, known as the Space of Disse. This buildup of ECM causes changes in the liver microarchitecture and can result in as much as a 6-fold increase in liver stiffness [6, 7].

Liver sinusoidal endothelial cells (LSECs) are specialized endothelial cells and play a critical role in maintaining normal liver homeostasis [2]. LSECs line the blood vessels in the liver forming a semi-permeable barrier between the blood and liver parenchyma [8, 9]. They play a vital role in the balance of lipids and cholesterol $[2,8,10]$. Through their scavenging properties, these cells eliminate components of connective tissues such as proteins and proteoglycans as well as debris from cell turnover [11]. LSECs exhibit pores called fenestrae that are approximately $100-200 \mathrm{~nm}$ in diameter [2, 9]. In LSECs, fenestrae enable the transport of nutrients and other molecules contained in blood 
between the sinusoidal lumen and the liver parenchyma. Under healthy conditions, LSECs do not exhibit the surface marker cluster of differentiation 31 (CD31) [12].

Associated with hepatic fibrosis is the process of capillarization, in which a complete basement membrane is formed underneath the sinusoidal endothelium, hindering the transport of macromolecules $[13,14]$. During capillarization, LSECs also experience a decrease in the size and number of fenestrae and eventually their complete disappearance $[15,16]$. Although, it is well known that the presence of fenestrae and their characteristics are reliant on the health of liver tissues, few studies have focused upon the relationship between LSECs and substrate rigidity [17]. There have been detailed investigations on how LSEC phenotype changes in response to being cultured on different types ECM [18, 19], however these studies do not elaborate on the mechanical properties of these ECM substrates. To the best of our knowledge, only one study has investigated the changes LSECs experience in response to differing rigidities [20]. Juin et al. examined the ability of LSECs to form podosomes (actin-rich protrusions involved in cell adhesion, migration, invasion and extracellular matrix degradation), when seeded on polyacrylamide hydrogels ranging in Young's Moduli from 1.75 to $20 \mathrm{kPa}$.

Critical to the progression of hepatic fibrosis, are the complex interactions between the parenchymal (hepatocytes) and non-parenchymal cells of the liver such as LSECs, Kupffer cells (KCs) and HSCs [3]. KCs, the resident macrophages of the liver, are known to secrete a number of inflammatory cytokines including tumor necrosis factor $\alpha$ (TNF- $\alpha$ ), transforming growth factor $\beta$ (TGF- $\beta$ ), interleukin $1 \alpha$ (IL-1 $\alpha$ ), and interleukin 6 (IL-6) [11]. The release of these cytokines can lead to the activation of HSCs, which in response secrete large amounts of ECM and continue the progression of fibrosis [1-3]. 
There have been reports in the literature that have shown the effects of other hepatic cell types, such as hepatocytes and HSCs, on LSEC phenotype [15, 19, 21, 22]. There are a few studies conducted in vivo that have examined how KCs may regulate LSEC behavior. It has been shown that in response to bile duct ligation in a rat model there is an increase in the number of KCs present in the liver correlating with an increase in the degree of fibrosis [23]. The study by Hutchins et al. [24] reported that in response to cecal ligation and puncture surgery to induce sepsis in mouse models KCs acted as potentiators of LSEC injury. The authors reported an increase in the interaction of programmed death receptor 1 (PDR1) on KCs with programmed death ligand 1 on LSECs leading to the decline of normal endothelial function. However, changes in fenestrae, signaling molecules and the effects of KCs were not investigated. To the best of our knowledge, there is no other study that has investigated the role of KCs on hLSEC behavior in a fibrotic microenvironment.

The onset and progression of liver fibrosis is a consequence of membrane rigidity, the secretion of inflammatory molecules and altered cellular signaling. In this study, we have examined the effect of matrix rigidity alone on phenotypic changes in human LSEC (hLSEC) monocultures. We have designed collagen gels exhibiting Young's moduli of 6 and $36 \mathrm{kPa}$ to closely mimic the stiffness found in a healthy and fibrotic liver, respectively $[6,7]$. To recapitulate the inflammatory microenvironment, we have cocultured hLSECs with rat KCs (rKCs). We report significant changes in hLSEC fenestrae, CD31 expression, actin organization and focal adhesions as well as in the levels of vascular endothelial growth factor (VEGF) and TNF- $\alpha$ as a result of a fibrotic and inflamed microenvironment.

\section{MATERIALS and METHODS}


2.1 Materials: Glutaraldehyde $(25 \% \mathrm{v} / \mathrm{v})$ was purchased from Electron Microscopy Sciences, Hatfield, PA. Bovine calf serum (BCS) was purchased from Hyclone, Logan, UT. Insulin was purchased from MP Biomedicals, Santa Ana, CA. Medium 199, endothelial cell growth supplement, L-glutamine, fetal bovine serum (FBS), penicillinstreptomycin, hexamethyldisilazane (HMDS), sodium-cacodylate, osmium tetroxide solution $(4 \% \mathrm{v} / \mathrm{v})$, chloroform, and $\beta$-mercaptoethanol were purchased from Sigma Aldrich, St. Louis, MO. Dulbecco's Modified Eagle Medium (DMEM) and phosphate buffered saline (PBS) were purchased from Invitrogen Life Technologies, Carlsbad, CA. All other chemicals and supplies were purchased from Thermo Fisher Scientific, Waltham, MA unless otherwise specified.

2.2 Casting collagen gels: Type I collagen solutions were diluted in 1X PBS to obtain solutions at two different concentrations. The final concentration of each solution was either 1.1 or $4.4 \mathrm{mg} / \mathrm{mL}$. Prior to casting gels, the diluted collagen solutions were mixed with 10X DMEM at a ratio of 9:1. The $\mathrm{pH}$ of collagen solutions was maintained between 6.9-7.0 for all experiments. Glass coverslips (18mm, Fisher Scientific) were activated using previously published procedures [25]. Briefly, coverslips were coated with $\mathrm{NaOH}$ and 3-aminopropyl tri-ethoxysilane for 10 minutes each and placed in glutaraldehyde $(8 \% \mathrm{v} / \mathrm{v})$ overnight. The activated coverslips were coated with collagen $(0.3 \mathrm{~mL} /$ coverslip) and incubated at $37^{\circ} \mathrm{C}$ for $1 \mathrm{~h}$ to promote crosslinking.

2.3 Profilometry: A Veeco Dektak 150 (Bruker, Billerica, MA) profiler was used to determine the hydrated thickness of the collagen gels. The thickness of the gels was determined by scanning across the gels at five different locations on three samples. The height of the gel was calculated by the difference in height between the surface of the gel and the underlying coverslip. Prior to taking measurements, the gels 
were placed in culture medium for a minimum of $2 \mathrm{~h}$ to ensure hydration. Thickness measurements were taken within 10 minutes of removing the gels from culture medium to prevent dehydration.

2.4 Atomic force microscopy (AFM): Young's modulus (YM) measurements of collagen hydrogels were obtained on hydrated samples using a Veeco MultiMode AFM (DNP-10, Veeco, Santa Barbara CA) equipped with a liquid cell chamber. The liquid cell chamber provided an enclosed environment with a watertight seal to enable measurements on hydrated samples. All measurements were conducted in contact mode using pyramidal SiN cantilever tips (Bruker AFM Probes, Camarillo, CA) with a spring constant of $0.06 \mathrm{Nm}^{-1}$ (minimum value $=0.03 \mathrm{Nm}^{-1}$, maximum value $=0.12 \mathrm{Nm}^{-1}$ ) . Force-distance curves were obtained at $1 \mathrm{~Hz}$ for a Z-scan distance of $1 \mu \mathrm{m}$ using blunted tips with a half open angle of $18^{\circ}$. The elastic modulus was obtained by fitting the raw data to a modified Hertz cone model using equations 1 and 2 [25].

(Equation 1)

$$
F=\frac{2 \text { tus } 4 x}{\pi}\left\lfloor\frac{L}{1-r^{2}}\right\rfloor \delta^{2}
$$

where $F=$ applied force, $\alpha=18^{\circ}, E=Y M, k=$ spring constant of the cantilever, $U=$ Poisson's ratio (constant $=0.25$ ) [26], $d=$ deflection of the cantilever, $d_{o}=$ deflection point during contact, and $\delta=$ indentation. Force distance curves were fit for indentations up to $10 \%$ of the overall hydrogel thickness to eliminate any contribution from the rigid glass coverslip.

2.5 Cell culture: hLSECs (ScienCell Research Laboratories, Carlsbad, CA) were maintained in Medium 199 supplemented with BCS (10\% v/v), penicillin-streptomycin $(1 \% \mathrm{v} / \mathrm{v})$, L-glutamine $(1 \% \mathrm{v} / \mathrm{v})$, and endothelial growth supplement $(2.5 \% \mathrm{w} / \mathrm{v}$, provided 
by the manufacturer). rKCs (Life Technologies) were maintained in DMEM supplemented with FCS $(10 \% \mathrm{v} / \mathrm{v})$, penicillin-streptomycin $(1 \% \mathrm{v} / \mathrm{v})$, insulin $(0.28 \% \mathrm{v} / \mathrm{v})$, and $\beta$-mercaptoethanol $(0.2 \% \mathrm{v} / \mathrm{v})$. hLSEC and rKC cultures were maintained for a minimum of $24 \mathrm{~h}$ prior to co-culture experiments. For monoculture experiments, hLSECs were seeded at a density of 30,000 cells per gel. For co-culture experiments, hLSECs were seeded with rKCs at ratios of 2:1 (30,000 hLSECs:15,000 rKCs) or 1:1 $(30,000$ hLSECs:30,000 rKCs) to mimic healthy and inflamed environments, respectively. Individual hLSEC and hLSEC-rKC co-cultures were maintained up to 4 days at $37^{\circ} \mathrm{C}$ in a humidified $5 \% \mathrm{CO}_{2}$ atmosphere. hLSECs were characterized by scanning electron microscopy (SEM) for fenestrae, immunofluorescence staining of CD31 expression, actin cytoskeleton, vinculin focal adhesions, and phase contrast microscopy to access cell proliferation and circularity. Levels of vascular endothelial growth factor (VEGF) and tumor necrosis factor a (TNF- $\alpha$ ) in spent media samples were assessed through enzyme linked immunosorbent analysis (ELISA) (Please refer to the supplementary information document for additional experimental information).

2.6 Statistical analysis: Statistical significance and $p$-values were calculated by a twosample $t$ test, assuming unequal variance. Statistical significance between different data sets was determined using ANOVA and compared post hoc using two tailed t-tests while applying the Bonferroni (multiple hypothesis testing) correction. For all statistical testing $\alpha=0.05$. All data are reported as mean \pm standard deviation; $n$ denotes sample size. 


\section{RESULTS}

\subsection{Effect of matrix rigidity on hLSEC phenotype:}

Collagen hydrogels exhibiting two different values for elastic modulus were prepared by varying the protein concentration in the gelling solutions. The difference in input protein concentration resulted in two substrates with distinctly different elastic moduli. The moduli of the gels measured by AFM were $6.0 \pm 2.3$ and $36 \pm 14.8 \mathrm{kPa}$ (Please refer to sample force distance curves in Supplementary Figure 1). The low and high values of elastic moduli were representative of elastic moduli reported for healthy and fibrotic liver tissues [7]. Collagen gel thicknesses measured by profilometry were found to be $1.77 \pm 0.24$ and $1.86 \pm 0.36 \mu \mathrm{m}$ for the $6 \mathrm{kPa}$ and $36 \mathrm{kPa}$ gels respectively.

Fenestrae arranged in sieve plates are observed in LSECs in vivo [27]. Their diameter and arrangement are indicative of the health and viability of hepatic tissues $[15,16,18]$. hLSECs seeded on $6 \mathrm{kPa}$ substrates exhibited well-defined fenestrae arranged in sieve plate structures (Figures 1A and 1B). The diameters and density of the fenestrae after $24 \mathrm{~h}$ were $140.7 \pm 52.6 \mathrm{~nm}(n=215)$, and $8-12 / \mu^{2} \quad$ (Supplementary Table 1). These values are similar to previous measurements of $100-200 \mathrm{~nm}$ in healthy human LSECs [9]. Upon culturing hLSECs for $96 \mathrm{~h}$ on the soft gels, the diameters and density of fenestrae decreased significantly to $66.9 \pm 21.6 \mathrm{~nm}(n=290)$ and $3-5 / \mu \mathrm{m}^{2}$, respectively. A dramatic difference was observed on $36 \mathrm{kPa}$ substrates, wherein hLSECs exhibited no fenestrae (Figure 1C). These trends clearly demonstrate that substrate rigidity and culture duration can lead to changes in fenestrae diameters and more importantly, can result in the complete disappearance of characteristic LSEC features [22, 28]. 
CD31, also known as platelet endothelial cell adhesion molecule, is present on most endothelial cells [29]. In their differentiated state, LSECs do not express this adhesion molecule on the surface $[30,31]$. However, CD31 can be expressed in de-differentiated or diseased LSECs [32, 33]. hLSECs cultured on 6kPa substrates did not exhibit CD31 after $24 \mathrm{~h}$ in culture (Figure 1D). However, this protein was expressed after 96h in culture (Figure 1E). CD31 was highly expressed in hLSECs cultured on a $36 \mathrm{kPa}$ substrate at the 24h time point (Figure 1F). The expression of CD31 expression has been shown to directly correlate with loss of fenestrae in LSECs [21] and together, these trends can be attributed to de-differentiation.

\subsection{Effect of Matrix Rigidity and rKCs on hLSEC Phenotype}

Although, matrix properties can affect LSEC phenotype, inflammation has also been shown to lead to a similar outcome [11, 24]. In order to test the effect of an inflammatory microenvironment, hLSEC-rKC co-cultures were investigated. hLSECs were co-cultured with $\mathrm{KCs}$ on both the $6 \mathrm{kPa}$ and $36 \mathrm{kPa}$ gels at ratios of 2 (hLSECs):1(rKCs) and 1(hLSECs):1(rKCs) representing healthy and inflamed microenvironments, respectively [23, 34]. In hLSEC:rKC co-cultures, changes in hLSEC phenotype were attributed to direct heterotypic cell-cell contact as well as the diffusion of soluble molecules (Figure 2A and 2B). First, changes in fenestrae were monitored upon the addition of rKCs. On $6 \mathrm{kPa}$ gels, at a 2:1 ratio, at the $24 \mathrm{~h}$ time-point, there was a significant decrease in fenestrae diameter and density (Figure 2C, Supplementary Table 1). Fenestrae diameters decreased to $57.1 \pm 19.5 \mu \mathrm{m}(n=310)$ and their density was $3-5 / \mu \mathrm{m}^{2}$ (Figure 2C). Upon increasing the number of rKCs to obtain a 1:1 ratio, fenestrae completely disappeared (Figure 2D). At 24h, on the stiffer substrate $(36 \mathrm{kPa})$ the addition of rKCs continued to result in a complete lack of fenestrae at 24h (Figures 2E and 2F). At 96h, 
no fenestrae were observed in either of the $\mathrm{rKC}$ co-cultures on the $6 \mathrm{kPa}$ substrates (Figures 2G and 2H).

VEGF is expressed by several hepatic cells such as hepatocytes, LSECs, KCs and HSCs [31, 35]. This growth factor has been shown to play a critical role in maintaining LSEC phenotype in vitro, specifically the presence of fenestrae $[21,22,36]$. We investigated VEGF levels in spent culture medium obtained from hLSEC monocultures and hLSEC-rKC co-cultures. The concentration of VEGF in unused culture medium was $4.0 \pm 1.0 \mathrm{pg} / \mathrm{mL}$. On the $6 \mathrm{kPa}$ gels, a statistically significant decrease $(p<0.05$ after applying the Bonferroni correction to values obtained with a 2 tailed $t$-test) in VEGF concentration was observed in two of the four co-cultures $(2: 1 \mathrm{hLSECs}: \mathrm{rKCs}$ at $24 \mathrm{~h}$ and 96h) when compared to LSECs monocultures at 24h (Figure 3A). However, there was no statistical difference in VEGF levels between LSEC monocultures (24h) and the cocultures that contained equal numbers of hLSECs and rKCs. On the $36 \mathrm{kPa}$ collagen gels, a statistically significant decrease in VEGF concentration was observed only in the 2:1 hLSEC:rKC (24h) co-culture (Figure 3B). Interestingly, a statistically higher concentration was observed in the 1:1 hLSEC:rKC (96h) co-culture. The decrease in VEGF levels correlate to the decrease in fenestrae diameter or their absence. Since KCs are known to express VEGF, the increase in concentration can be attributed to the presence of these resident hepatic macrophages [31]. Despite this increase in VEGF levels, hLSECs in this co-culture did not exhibit fenestrae, thereby, suggesting the role of other signaling molecules that may play a role in controlling the presence or absence of fenestrae (see Discussion).

To differentiate between rKCs and hLSECs in co-cultures, a dual immunofluorescence staining was conducted. Antibodies to CD163 (rKCs only) and CD31 (hLSECs and 
rKCs) were added (Please refer to Supplementary Figure 2). KCs have previously been reported to express CD31 $[37,38]$. In the co-cultures, rKCs were clearly identified by the presence of CD163. CD31 expression on the surface of hLSECs also changed upon the addition of rKCs. Weak green fluorescence indicative of CD31 expression was observed in the 2:1 hLSEC:rKC co-cultures with an increase in fluorescence intensity in the 1:1 hLSEC:rKC co-cultures on the 6kPa gels (Figures 4A and 4B). CD31 was highly expressed in hLSECs co-cultured with rKCs on the 36kPa substrates (Figures $4 \mathrm{C}$ and 4D) and on the $6 \mathrm{kPa}$ gels at $96 \mathrm{~h}$ (Figures $4 \mathrm{E}$ and $4 \mathrm{~F}$ ). Together, these trends clearly illustrate the effect of rKCs on hLSEC phenotype. Since CD31 expression was observed even on the $6 \mathrm{kPa}$ gels in the presence of rKCs, the presence of cytokines or other signaling molecules secreted by rKCs appear to be exerting a stronger effect on hLSECs than substrate rigidity alone.

Changes in the organization of the actin cytoskeleton have been linked to decreases in fenestrae diameter and in some cases, the absence of such pores [39]. Therefore, we investigated changes in f-actin distribution and vinculin focal adhesion to determine their effect on fenestrae. In hLSEC monocultures, matrix rigidity and the duration in culture influenced f-actin organization. On $6 \mathrm{kPa}$ gels at $24 \mathrm{~h}$, diffuse actin fibers were observed in hLSECs, in contrast to well defined stress fibers on a stiffer matrix (36kPa) (Figures 5A and 5B). Similarly, a $96 \mathrm{~h}$ culture on the $6 \mathrm{kPa}$ gels resulted in f-actin stress (Supplementary Figure 3A). These trends correlate to the loss of fenestrae on stiffer substrates and with longer culture periods. When hLSECs were co-cultured with rKCs at a 2:1 ratio, f-actin stress fibers were well organized only in hLSECs on the $36 \mathrm{kPa}$ gels or at the 96h time-point on 6kPa gels (Figures 5C and 5D, Supplementary Figure 3B). At a 1:1 ratio of hLSECs-rKCs, actin fibers were visible under all conditions (Figures 5E and 5F, Supplementary Figure 3C). A similar trend was observed with vinculin focal 
adhesions. Stiffer matrices resulted in larger adhesion sites in hLSEC monocultures (Figures 5A and 5B). In hLSEC monocultures, the diameters of vinculin focal adhesions were approximately two-fold higher on the $36 \mathrm{kPa}\left(1.44 \pm 0.66 \mu \mathrm{m}^{2} n=60\right)$ in comparison to those observed on $6 \mathrm{kPa}$ gels $\left(0.69 \pm 0.31 \mu \mathrm{m}^{2} n=50\right)$ (Table 1). The inclusion of rKCs to hLSEC monocultures (6kPa gels) resulted in greater focal adhesion areas wherein the increase was statistically significant. The focal adhesions were $1.07 \pm 0.47$ and $1.42 \pm$ $0.43 \mu \mathrm{m}^{2}$ for the $2: 1$ and $1: 1$ ratios respectively. A similar trend was observed for cells cultured on the soft matrices at $96 \mathrm{~h}$. However, focal adhesion size did not change for hLSECs adherent on the $36 \mathrm{kPa}$ substrates suggesting that signaling molecules secreted by rKCs could not enhance the effect of matrix rigidity alone.

The differences in actin organization on the softer gels upon the addition of rKCs were the result of cytokine-mediated signaling. KCs secrete cytokines such as IL-1a, IL-6, TGF- $\beta$, and TNF- $\alpha$ [11]. Of these cytokines, TNF- $\alpha$ has been implicated in the reorganization of the actin cytoskeleton in endothelial cells [40]. Although, LSECs can secrete TNF- $\alpha$, the levels are significantly lower in comparison to KCs $[41,42]$. The concentration of TNF- $\alpha$ was measured in the spent culture medium of all samples. In our investigations, the concentration of this cytokine was influenced by culture timeperiod and the presence of rKCs. At the $24 \mathrm{~h}$ time-point, this cytokine was produced only at the $1: 1$ ratio on both gels for hLSEC-rKC co-cultures and at the $2: 1$ ratio on the $36 \mathrm{kPa}$ gels (Figure 6A). All other conditions did not produce detectable levels of TNF- $\alpha$. When the cultures were maintained for 96h, all conditions with the exception of hLSEC monocultures (36kPa) secreted TNF- $\alpha$ (Figure 6B). On soft collagen gels $(6 \mathrm{kPa})$, there was no difference in TNF- $\alpha$ concentration even upon increasing the number of rKCs. In contrast, a 2-fold (statistically significant, $p=1.3 \times \mathrm{E}$-05) increase in TNF- $\alpha$ was observed upon increasing the number of rKCs in co-cultures on the $36 \mathrm{kPa}$ gels. 
Matrix elasticity also influenced the proliferation and morphology of hLSECs (Figure 7, Supplementary Table 2). While hLSECs proliferation was higher on soft (6kPa) gels, a reverse trend was observed with rKCs. When seeded on the $6 \mathrm{kPa}$ gels, hLSECs exhibited a 2.1 fold increase in cell number (Figure 7A) while their number remained virtually unchanged with the addition of rKCs on the $6 \mathrm{kPa}$ gels (Figure 7B) and in monocultures on the $36 \mathrm{kPa}$ gels (Figure 7C). Interestingly, rKCs exhibited higher proliferation on stiffer gels corresponding to 3.45 and 3.17 fold increases when seeded at 2:1 and 1:1 ratios. The addition of rKCs resulted in lower hLSEC proliferation rates in all co-cultures (Supplementary Table 2).

Projected cell areas and circularity were measured for both hLSECs and rKCs $(n \geq 150)$. An approximate two-fold increase was seen in hLSEC area on the $36 \mathrm{kPa}$ gels in comparison to cells on the $6 \mathrm{kPa}$ gels (Table 2). The projected cell areas for hLSECs were observed to be approximately $25-65$ fold higher than rKCs in each of the cocultures. Interestingly, increased hLSEC areas could be correlated to the presence of rKCs. Cell circularity was assessed as a measure of cell elongation and the formation of protrusions. hLSECs seeded on the $36 \mathrm{kPa}$ gels exhibited circularity values significantly lower than those seeded on the $6 \mathrm{kPa}$ gels, due to their elongated shape corresponding to circularities of $0.25 \pm 0.12$ and $0.32 \pm 0.14$ (Figure 7D) respectively (Table 2). The elongation of the LSECs on the stiffer substrates as well as the formation of long, branching protrusions is suggestive of capillarization. As with cell area, the addition of rKCs to the $6 \mathrm{kPa}$ gels resulted in a statistically significant change in cell circularity. hLSEC circularities of $0.26 \pm 0.10$ and $0.27 \pm 0.11$ were observed for the $2: 1$ and $1: 1$ ratios respectively on the $6 \mathrm{kPa}$ gels. No significant change was found when rKCs were added to the $36 \mathrm{kPa}$ gels resulting in hLSEC circularities of $0.26 \pm 0.10$ and $0.24 \pm 0.09$ 
(Figure 7E) for the $2: 1$ and $1: 1$ ratios respectively. rKC circularities were significantly higher (approximately 0.9 ) than those of hLSECs for all co-cultures.

\section{CONCLUSIONS and DISCUSSION}

Liver fibrosis can result due to several health conditions. As a consequence, liver cells secrete and deposit excessive amounts of ECM proteins such as proteoglycans, glycosaminoglycans, and matrix-tethered growth factors, which in turn lead to altered cellular phenotypes and perturbed function [1,3]. Changes in ECM rigidity due to the deposition of these proteins leads to the transformation of HSCs from a "quiescent" to an "activated" state. This critical event is necessary for the progression of fibrosis. Upon activation, HSCs secrete more ECM proteins and inflammatory molecules such as cytokines. This process leads to hepatocytes undergoing inflammation and then apoptosis, leading to the recruitment of KCs. KCs in turn secrete pro-inflammatory cytokines that continue to alter cell signaling. This complex interplay between matrix rigidity and inflammatory cues enables the progression of fibrosis.

In this study, we have demonstrated significant changes in hLSECs that occur solely due to the elasticity of their underlying substrate. A significant finding was that loss of fenestrae, expression of surface CD31 and well-organized actin cytoskeleton could occur even in cells adherent on soft substrates when KCs are added. This suggests that signaling molecules secreted by KCs can undermine the effects of a soft matrix that is representative of healthy tissues.

We investigated the levels of VEGF since this growth factor stimulates the production of endothelial nitric oxide synthase (eNOS), promoting the LSEC phenotype [21]. In 
cirrhotic rat livers, the levels of eNOS have been shown to be significantly less than those found in healthy livers $[43,44]$. The presence of $r K C s$ resulted in significant reductions in VEGF secreted by hLSECs even on 6kPa collagen gels. The decrease in VEGF was accompanied by a concomitant increase in actin stress fibers. The actin cytoskeleton has been shown to play a crucial role in the maintenance and formation of LSEC fenestrae $[39,45,46]$. The reorganization of the actin cytoskeleton occurs through a hierarchical cascade of Rho-GTPase proteins including cell division cycle 42 (Cdc42), Rac, and Rho [47]. Treatment of endothelial cells with TNF- $\alpha$ has also been shown to result in a shift from g-actin to f-actin and larger traction forces exerted by the cells [48], as well as the phosphorylation of paxillin and focal adhesion kinase resulting in larger focal adhesions [49]. Taken together, these studies suggest that TNF- $\alpha$ may be responsible for the changes observed in the cytoskeletal structure as well as fenestrae on hLSECs when seeded with rKCs on the $6 \mathrm{kPa}$ gels. Force transmitted through the actin cytoskeleton through Rho-mediated contractility results in an increased force applied to the substrate, which further promotes focal adhesion assembly [50]. This increase in focal adhesion size and formation of actin stress fibers is indicative of a higher contractility in hLSECs seeded on the $36 \mathrm{kPa}$ substrates. It has previously been shown that ligand density can also affect cytoskeletal dynamics both in tandem with and independently of substrate stiffness [51]. Ligand density, as well as substrate viscoelasticity will change with increasing collagen fiber density. Therefore, in future studies, it would be beneficial to further separate the effects of the substrate stiffness from collagen fiber density. This will enable understanding the role of fiber density on the actin cytoskeleton and fenestrae organization.

To the best of our knowledge this is the first study that has investigated the effects of KCs on hLSEC phenotype on soft materials. Our studies clearly indicate that cytokine 
secretion by rKCs can overcome any beneficial effects of a soft substrate. These studies provide insights into the onset and progression of hepatic fibrosis as a function of a transitioning microenvironment.

\section{ACKNOWLEDGEMENTS}

We gratefully acknowledge financial support from the National Science Foundation (NSF DMR 0955873 and DMR 1410341). We also acknowledge financial support from the Institute for Critical Technologies and Applied Sciences at Virginia Tech. 


\section{REFERENCES}

[1] Grigorescu M. Noninvasive biochemical markers of liver fibrosis. J Gastrointestin Liver Dis 2006;15:149-59.

[2] Arias IM, Boyer JL, Chisari FV, Fausto M, Schachter D, Shafritz DA. The liver: biology and pathobiology. Lippincott Williams and Wilkins; 2001.

[3] Bataller R, Brenner DA. Liver fibrosis. J Clin Invest 2005;115:209-18.

[4] Rauterberg J, Voss B, Pott G, Gerlach U. Connective tissue components of the normal and fibrotic liver. I. Structure, local distribution and metabolism of connective tissue components in the normal liver and changes in chronic liver diseases. Klin Wochenschr 1981;59:767-79.

[5] Georges PC, Hui JJ, Gombos Z, McCormick ME, Wang AY, Uemura M, et al. Increased stiffness of the rat liver precedes matrix deposition: implications for fibrosis. American journal of physiology Gastrointestinal and liver physiology 2007;293:G1147-54.

[6] Wong VWS, Vergniol J, Wong GLH, Foucher J, Chan HLY, Le Bail B, et al. Diagnosis of Fibrosis and Cirrhosis Using Liver Stiffness Measurement in Nonalcoholic Fatty Liver Disease. Hepatology 2010;51:454-62.

[7] Mueller S, Sandrin L. Liver stiffness: a novel parameter for the diagnosis of liver disease. Hepatic medicine : evidence and research 2010;2:49-67.

[8] Wisse E, Braet F, Dianzhong I. Endothelial cells of the hepatic sinusoids: a review. liver diseases and hepatic sinusoidal cells. Tokyo: Springer-Verlag; 1999.

[9] Braet F, Wisse E. Structural and functional aspects of liver sinusoidal endothelial cell fenestrae: a review. Comparative hepatology 2002;1:1.

[10] Smedsrod B. Clearance function of scavenger endothelial cells. Comp Hepatol 2004;3 Suppl 1:S22.

[11] Smedsrod B, Debleser PJ, Braet F, Lovisetti P, Vanderkerken K, Wisse E, et al. Cell Biology of Liver Endothelial and Kupffer Cells. Gut 1994;35:1509-16.

[12] DeLeve LD, Wang XD, McCuskey MK, McCuskey RS. Rat liver endothelial cells isolated by anti-CD31 immunomagnetic separation lack fenestrae and sieve plates. Am J Physiol-Gastr L 2006;291:G1187-G9.

[13] Xu B, Broome U, Uzunel M, Nava S, Ge XP, Kumagai-Braesch M, et al. Capillarization of hepatic sinusoid by liver endothelial cell-reactive autoantibodies in patients with cirrhosis and chronic hepatitis. Am J Pathol 2003;163:1275-89. [14] Xu GF, Wang XY, Ge GL, Li PT, Jia X, Tian DL, et al. Dynamic changes of capillarization and peri-sinusoid fibrosis in alcoholic liver diseases. World J Gastroentero 2004;10:238-43.

[15] Xie GH, Wang XD, Wang L, Wang L, Atkinson RD, Kanel GC, et al. Role of Differentiation of Liver Sinusoidal Endothelial Cells in Progression and Regression of Hepatic Fibrosis in Rats. Gastroenterology 2012;142:918-U392.

[16] DeLeve LD, Wang X, Kanel GC, Atkinson RD, McCuskey RS. Prevention of hepatic fibrosis in a murine model of metabolic syndrome with nonalcoholic steatohepatitis. Am J Pathol 2008;173:993-1001. 
[17] Wells RG. The role of matrix stiffness in regulating cell behavior. Hepatology 2008;47:1394-400.

[18] Sellaro TL, Ravindra AK, Stolz DB, Badylak SF. Maintenance of hepatic sinusoidal endothelial cell phenotype in vitro using organ-specific extracellular matrix scaffolds. Tissue Eng 2007;13:2301-10.

[19] March S, Hui EE, Underhill GH, Khetani S, Bhatia SN. Microenvironmental Regulation of the Sinusoidal Endothelial Cell Phenotype In Vitro. Hepatology 2009;50:920-8.

[20] Juin A, Planus E, Guillemot F, Horakova P, Albiges-Rizo C, Genot E, et al. Extracellular matrix rigidity controls podosome induction in microvascular endothelial cells. Biology of the cell / under the auspices of the European Cell Biology Organization 2013;105:46-57.

[21] DeLeve LD, Wang XD, Hu LP, McCuskey MK, McCuskey RS. Rat liver sinusoidal endothelial cell phenotype is maintained by paracrine and autocrine regulation. Am J Physiol-Gastr L 2004;287:G757-G63.

[22] Krause P, Markus PM, Schwartz P, Unthan-Fechner K, Pestel S, Fandrey J, et al. Hepatocyte-supported serum-free culture of rat liver sinusoidal endothelial cells.

Journal of hepatology 2000;32:718-26.

[23] Grinko I, Geerts A, Wisse E. Experimental biliary fibrosis correlates with increased numbers of fat-storing and Kupffer cells, and portal endotoxemia. Journal of hepatology 1995;23:449-58.

[24] Hutchins NA, Wang F, Wang Y, Chung CS, Ayala A. Kupffer cells potentiate liver sinusoidal endothelial cell injury in sepsis by ligating programmed cell death ligand1. J Leukocyte Biol 2013;94:963-70.

[25] Hale NA, Yang Y, Rajagopalan P. Cell Migration at the Interface of a Dual Chemical-Mechanical Gradient. ACS Applied Materials \& Interfaces 2010;2:2317-24. [26] Barocas VH, Moon AG, Tranquillo RT. The Fibroblast-Populated Collagen Microsphere Assay of Cell Traction Force .2. Measurement of the Cell Traction Parameter. J Biomech Eng-T Asme 1995;117:161-70.

[27] Wisse E. An electron microscopic study of the fenestrated endothelial lining of rat liver sinusoids. Journal of ultrastructure research 1970;31:125-50.

[28] Hang TC, Lauffenburger DA, Griffith LG, Stolz DB. Lipids promote survival, proliferation, and maintenance of differentiation of rat liver sinusoidal endothelial cells in vitro. American journal of physiology Gastrointestinal and liver physiology 2012;302:G375-88.

[29] Albelda SM, Muller WA, Buck CA, Newman PJ. Molecular and cellular properties of PECAM-1 (endoCAM/CD31): a novel vascular cell-cell adhesion molecule. The Journal of cell biology 1991;114:1059-68.

[30] Scoazec JY, Feldmann G. The cell adhesion molecules of hepatic sinusoidal endothelial cells. Journal of hepatology 1994;20:296-300.

[31] Hwa A, Fry R, Sivaraman A, So P, Samson L, Stolz D, et al. Rat liver sinusoidal endothelial cells survive without exogenous VEGF in 3D perfused co-cultures with hepatocytes. The FASEB journal 2007;21:2564-79.

[32] Couvelard A, Scoazec JY, Feldmann G. Expression of cell-cell and cell-matrix adhesion proteins by sinusoidal endothelial cells in the normal and cirrhotic human liver. Am J Pathol 1993;143:738-52. 
[33] Scoazec JY, Flejou JF, D'Errico A, Couvelard A, Kozyraki R, Fiorentino M, et al. Focal nodular hyperplasia of the liver: composition of the extracellular matrix and expression of cell-cell and cell-matrix adhesion molecules. Human pathology 1995;26:1114-25.

[34] Mehal WZ, Azzaroli F, Crispe IN. Immunology of the healthy liver: old questions and new insights. Gastroenterology 2001;120:250-60.

[35] Kawasaki T, Murata S, Takahashi K, Nozaki R, Ohshiro Y, Ikeda N, et al. Activation of human liver sinusoidal endothelial cell by human platelets induces hepatocyte proliferation. Journal of hepatology 2010;53:648-54.

[36] Deleve LD, Wang X, Guo Y. Sinusoidal endothelial cells prevent rat stellate cell activation and promote reversion to quiescence. Hepatology 2008;48:920-30.

[37] Neubauer K, Lindhorst A, Tron K, Ramadori G, Saile B. Decrease of PECAM-1gene-expression induced by proinflammatory cytokines IFN-gamma and IFN-alpha is reversed by TGF-beta in sinusoidal endothelial cells and hepatic mononuclear phagocytes. BMC physiology 2008;8:9.

[38] Lalor PF, Lai WK, Curbishley SM, Shetty S, Adams DH. Human hepatic sinusoidal endothelial cells can be distinguished by expression of phenotypic markers related to their specialised functions in vivo. World journal of gastroenterology : WJG 2006;12:5429-39.

[39] Yokomori H, Yoshimura K, Funakoshi S, Nagai T, Fujimaki K, Nomura M, et al. Rho modulates hepatic sinusoidal endothelial fenestrae via regulation of the actin cytoskeleton in rat endothelial cells. Laboratory investigation; a journal of technical methods and pathology 2004;84:857-64.

[40] Mathew SJ, Haubert D, Kronke M, Leptin M. Looking beyond death: a morphogenetic role for the TNF signalling pathway. Journal of cell science 2009;122:1939-46.

[41] Gulubova MV. Expression of cell adhesion molecules, their ligands and tumour necrosis factor alpha in the liver of patients with metastatic gastrointestinal carcinomas. The Histochemical journal 2002;34:67-77.

[42] Yamashita M, Taniyama M, Tamai M. Cellular localization of tumor necrosis factor-alpha mRNA and interleukin-6 mRNA in the rat liver after hemorrhagic shock. Surgery today 2002;32:701-6.

[43] Shah V, Toruner M, Haddad F, Cadelina G, Papapetropoulos A, Choo K, et al. Impaired endothelial nitric oxide synthase activity associated with enhanced caveolin binding in experimental cirrhosis in the rat. Gastroenterology 1999;117:1222-8.

[44] Rockey DC, Chung JJ. Reduced nitric oxide production by endothelial cells in cirrhotic rat liver: endothelial dysfunction in portal hypertension. Gastroenterology 1998;114:344-51.

[45] Braet F, Spector I, Shochet N, Crews P, Higa T, Menu E, et al. The new anti-actin agent dihydrohalichondramide reveals fenestrae-forming centers in hepatic endothelial cells. BMC cell biology 2002;3:7.

[46] Braet F. Rac1, caveolin-1 and vascular endothelial growth factor -mediated liver sinusoidal endothelial cell angiogenesis. Liver international : official journal of the International Association for the Study of the Liver 2009;29:143-4. 
[47] Wojciak-Stothard B, Entwistle A, Garg R, Ridley AJ. Regulation of TNF-alphainduced reorganization of the actin cytoskeleton and cell-cell junctions by Rho, Rac, and Cdc42 in human endothelial cells. J Cell Physiol 1998;176:150-65.

[48] Stroka KM, Vaitkus JA, Aranda-Espinoza H. Endothelial cells undergo morphological, biomechanical, and dynamic changes in response to tumor necrosis factor-alpha. European biophysics journal : EBJ 2012;41:939-47.

[49] Koukouritaki SB, Vardaki EA, Papakonstanti EA, Lianos E, Stournaras C, Emmanouel DS. TNF-alpha induces actin cytoskeleton reorganization in glomerular epithelial cells involving tyrosine phosphorylation of paxillin and focal adhesion kinase. Mol Med 1999;5:382-92.

[50] Provenzano PP, Keely PJ. Mechanical signaling through the cytoskeleton regulates cell proliferation by coordinated focal adhesion and Rho GTPase signaling. Journal of cell science 2011;124:1195-205.

[51] Engler A, Bacakova L, Newman C, Hategan A, Griffin M, Discher D. Substrate compliance versus ligand density in cell on gel responses. Biophys J 2004;86:61728. 


\section{FIGURE CAPTIONS}

Figure 1. SEM images of hLSECs seeded on collagen gels. (A) hLSECs seeded on the $6 \mathrm{kPa}$ gels for $24 \mathrm{~h}$ showed well defined fenestrae arranged in sieve plates, indicated by the white arrows. (B) After $96 \mathrm{~h}$ on the $6 \mathrm{kPa}$ gels, fenestrae size decreased in size. (C) After $24 \mathrm{~h}$ on the $36 \mathrm{kPa}$ gels, hLSECs showed a complete lack of fenestrae. Inserts in the top right corner of each panel depict the entire cell. Immunofluorescence images of CD31 surface expression for (D) hLSECs seeded on the 6kPa gels for 24h, (E) hLSECs at $96 \mathrm{~h}$ on $6 \mathrm{kPa}$ gels, $(\mathrm{F}) \mathrm{hLSECs}$ at $24 \mathrm{~h}$ on the $36 \mathrm{kPa}$ gels. Scale bars $=5 \mu \mathrm{m}(\mathrm{A}-\mathrm{C})$, scale bars $=100 \mu \mathrm{m}(\mathrm{D}-\mathrm{F})$.

Figure 2. SEM images of hLSEC surfaces when co-cultured with rKCs. hLSECs cocultured with rKCs at ratios of (A) 2:1 and (B) 1:1 on $6 \mathrm{kPa}$ gels at $24 \mathrm{~h}$. hLSECs are indicated by black arrows and rKCs by white arrows. (C) Fenestrae in hLSECs cultured at a 2:1 ratio with rKCs on the $6 \mathrm{kPa}$ gels at $24 \mathrm{~h}$, (D) A complete lack of fenestrae on hLSECs seeded at a 1:1 ratio with rKCs on the $6 \mathrm{kPa}$ gels after $24 \mathrm{~h},(\mathrm{E})$ a 2:1 ratio with rKCs on the $36 \mathrm{kPa}$ gels after $24 \mathrm{~h},(\mathrm{~F})$ a 1:1 ratio with rKCs on the $36 \mathrm{kPa}$ gels after $96 \mathrm{~h}$, (G) a 2:1 ratio with rKCs on the $6 \mathrm{kPa}$ gels after $96 \mathrm{~h}$, and $(\mathrm{H})$ a 1:1 ratio with $\mathrm{rKCs}$ on the $6 \mathrm{kPa}$ gels after $96 \mathrm{~h}$. Scale bars $=5 \mu \mathrm{m}(\mathrm{A}-\mathrm{B})$. Scale bars $=5 \mu \mathrm{m}(\mathrm{C}-\mathrm{H})$.

Figure 3. VEGF expression in spent media samples from hLSEC monocultures and hLSEC-rKC co-cultures on (A) $6 \mathrm{kPa}$ and (B) $36 \mathrm{kPa}$ gels as determined by ELISA. An asterisk $\left(^{*}\right)$ indicates a statistically significant difference from hLSEC monocultures at 24h. Statistical significance was determined by a 2 tailed $\mathrm{t}$-test after applying the Bonferroni correction to $p$-values with $\alpha=0.05$. 
Figure 4. Immunofluorescence images of surface CD31 expression of hLSECs when co-cultured with rKCs. (A-B) An increase in hLSEC CD31 expression is observed after $24 \mathrm{~h}$ when co-cultured with rKCs at ratios of $2: 1$ and $1: 1$ on the $6 \mathrm{kPa}$ gels. (C-D) High levels of CD31 expression are observed at $24 \mathrm{~h}$ on hLSECs cultured on the $36 \mathrm{kPa}$ gels with rKCs at ratios of 2:1 and 1:1. (E-F) After 96h on the 6kPa gels, hLSECs exhibit increased amounts of CD31 expression when seeded at ratios of 2:1 and 1:1 with rKCs. Scale bars $=100 \mu \mathrm{m}$.

Figure 5. Immunofluorescence images of actin cytoskeleton (red) and vinculin focal adhesions (green) within hLSEC monocultures and hLSEC-rKC co-cultures at $24 \mathrm{~h}$. (A) When seeded alone on the $6 \mathrm{kPa}$ gels, diffuse actin fibers were observed as well as small focal adhesions. (B) hLSECs exhibit thick actin stress fibers when seeded on the $36 \mathrm{kPa}$ gels at $24 \mathrm{~h}$. At the $2: 1 \mathrm{hLSEC}-\mathrm{rKC}$ ratio on the $6 \mathrm{kPa}$ gels $(\mathrm{C})$, actin structure was similar to that of the monocultures. Well-organized actin stress fibers were seen in hLSECs on the $36 \mathrm{kPa}$ at the $2: 1 \mathrm{hLSEC}-\mathrm{rKC}$ ratio (D). Thick actin stress fibers were observed at the 1:1 hLSEC-rKC ratio for both the $(\mathrm{E}) 6 \mathrm{kPa}$ and $(\mathrm{F}) 36 \mathrm{kPa}$ gels. Regions with well-defined actin stress fibers are indicated by an asterisk $\left(^{*}\right)$. Scale bars $=50 \mu \mathrm{m}$.

Figure 6. TNF- $\alpha$ expression in spent media samples from hLSEC monocultures and hLSEC-rKC co-cultures at (A) 24h and (B) 96h as determined by ELISA. An asterisk ( $\left.{ }^{*}\right)$ indicates a statistically significant difference from the LSEC monocultures while a number sign (\#) indicates a statistically significant difference between co-culture ratios. Conditions indicated by a cross $(\dagger)$ did not show any detectable levels of TNF- $\alpha$. Statistical significance was determined by a 2 tailed t-test, applying the Bonferroni correction to $p$-values when multiple comparisons were made, with $\alpha=0.05$. 
Figure 7. Merged phase contrast and fluorescence images of a (A) 6kPa hLSEC monoculture, (B) 6kPa 1:1 hLSEC-rKC co-culture, and (C) 36kPa hLSEC monoculture at 96h showing a decrease in hLSEC proliferation with an increase in substrate stiffness or addition of rKCs. hLSECs were labeled with a red cytoplasmic dye prior to seeding on the collagen gels. Representative cells for (D) 6kPa hLSEC monocultures and (E) 36kPa 1:1 hLSEC-rKC co-cultures having cell circularities of 0.39 and 0.19 respectively. Scale bars $=50 \mu \mathrm{m}$. 
Table 1: Focal adhesion areas in hLSEC and hLSEC:rKC cultures.

\begin{tabular}{|c|c|c|c|c|}
\hline $\begin{array}{c}\text { Gel YM } \\
(\mathbf{k P a})\end{array}$ & Cell Culture & $\begin{array}{c}\text { Time Point } \\
\mathbf{( h )}\end{array}$ & $\begin{array}{c}\text { Focal Adhesion } \\
\text { Area } \\
\left(\mathbf{\mu m}^{2}\right)\end{array}$ & $\begin{array}{c}\boldsymbol{n} \\
\text { (Focal } \\
\text { Adhesions) }\end{array}$ \\
\hline 6 & $\begin{array}{c}\text { hLSEC } \\
\text { Monocultures }\end{array}$ & 24 & $0.69 \pm 0.31$ & 50 \\
\hline 6 & $\begin{array}{c}2: 1 \\
\text { hLSEC:rKC }\end{array}$ & 24 & $1.07 \pm 0.47$ & 110 \\
\hline 6 & $\begin{array}{c}1: 1 \\
\text { hLSEC:rKC }\end{array}$ & 24 & $1.42 \pm 0.43$ & 70 \\
\hline 36 & $\begin{array}{c}\text { hLSEC } \\
\text { Monocultures }\end{array}$ & 24 & $1.44 \pm 0.66$ & 60 \\
\hline 36 & $\begin{array}{c}2: 1 \\
\text { hLSEC:rKC }\end{array}$ & 24 & $1.45 \pm 0.38$ & 60 \\
\hline 36 & $\begin{array}{c}1: 1 \\
\text { hLSEC:rKC }\end{array}$ & 24 & $1.51 \pm 0.42$ & 100 \\
\hline 6 & $\begin{array}{c}\text { hLSEC } \\
\text { Monocultures }\end{array}$ & 96 & $0.78 \pm 0.22$ & 100 \\
\hline 6 & $\begin{array}{c}2: 1 \\
\text { hLSEC:rKC }\end{array}$ & 96 & $1.27 \pm 0.38$ & 100 \\
\hline 6 & $\begin{array}{c}1: 1 \\
\text { hLSEC:rKC }\end{array}$ & 96 & $1.34 \pm 0.35$ & 50 \\
\hline
\end{tabular}


Table 2: hLSEC and rKC morphology ( $\mathrm{n} \geq 150)$

\begin{tabular}{|c|c|c|c|c|c|}
\hline $\begin{array}{c}\text { Gel } \\
\text { YM } \\
(\mathbf{k P a})\end{array}$ & Cell Culture & $\begin{array}{c}\text { hLSEC } \\
\text { Area } \\
\left(\boldsymbol{\mu m}^{2}\right)\end{array}$ & $\begin{array}{c}\mathbf{r K C} \\
\text { Area } \\
\left(\boldsymbol{\mu \mathbf { m } ^ { 2 } )}\right.\end{array}$ & $\begin{array}{c}\text { hLSEC } \\
\text { Circularity }\end{array}$ & $\begin{array}{c}\mathbf{r K C} \\
\text { Circularity }\end{array}$ \\
\hline 6 & $\begin{array}{c}\text { hLSEC } \\
\text { Monocultures }\end{array}$ & $\begin{array}{c}1600 \pm \\
980\end{array}$ & 0 & $0.32 \pm 0.14$ & 0 \\
\hline 6 & $\begin{array}{c}2: 1 \\
\text { hLSEC:rKC }\end{array}$ & $\begin{array}{c}3100 \pm \\
2600\end{array}$ & $\begin{array}{c}52.2 \pm \\
33.4\end{array}$ & $0.26 \pm 0.10$ & $0.89 \pm 0.07$ \\
\hline 6 & $\begin{array}{c}1: 1 \\
\text { hLSEC:rKC }\end{array}$ & $\begin{array}{c}3120 \pm \\
2680\end{array}$ & $\begin{array}{c}52.0 \pm \\
30.4\end{array}$ & $0.27 \pm 0.11$ & $0.89 \pm 0.08$ \\
\hline 36 & $\begin{array}{c}\text { hLSEC } \\
\text { Monocultures }\end{array}$ & $\begin{array}{c}3070 \pm \\
1670\end{array}$ & 0 & $0.25 \pm 0.12$ & 0 \\
\hline 36 & $\begin{array}{c}2: 1 \\
\text { hLSEC:rKC }\end{array}$ & $\begin{array}{c}2720 \pm \\
1860\end{array}$ & $\begin{array}{c}66.3 \pm \\
30.4\end{array}$ & $0.26 \pm 0.10$ & $0.90 \pm 0.06$ \\
\hline 36 & $\begin{array}{c}1: 1 \\
\text { hLSEC:rKC }\end{array}$ & $\begin{array}{c}2740 \pm \\
1760\end{array}$ & $\begin{array}{c}58.2 \pm \\
28.4\end{array}$ & $0.24 \pm 0.09$ & $0.89 \pm 0.07$ \\
\hline
\end{tabular}



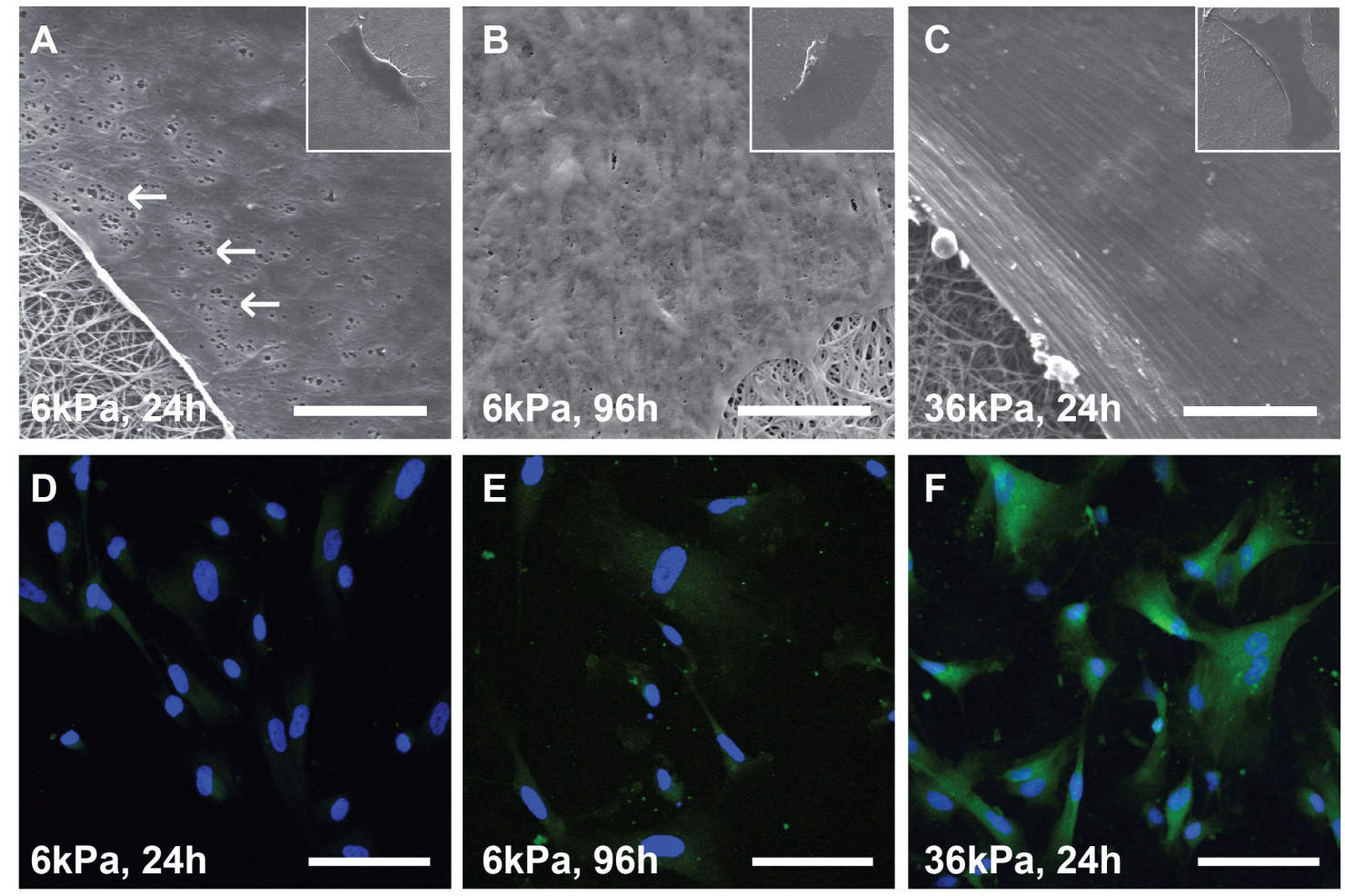

Fig. 1 

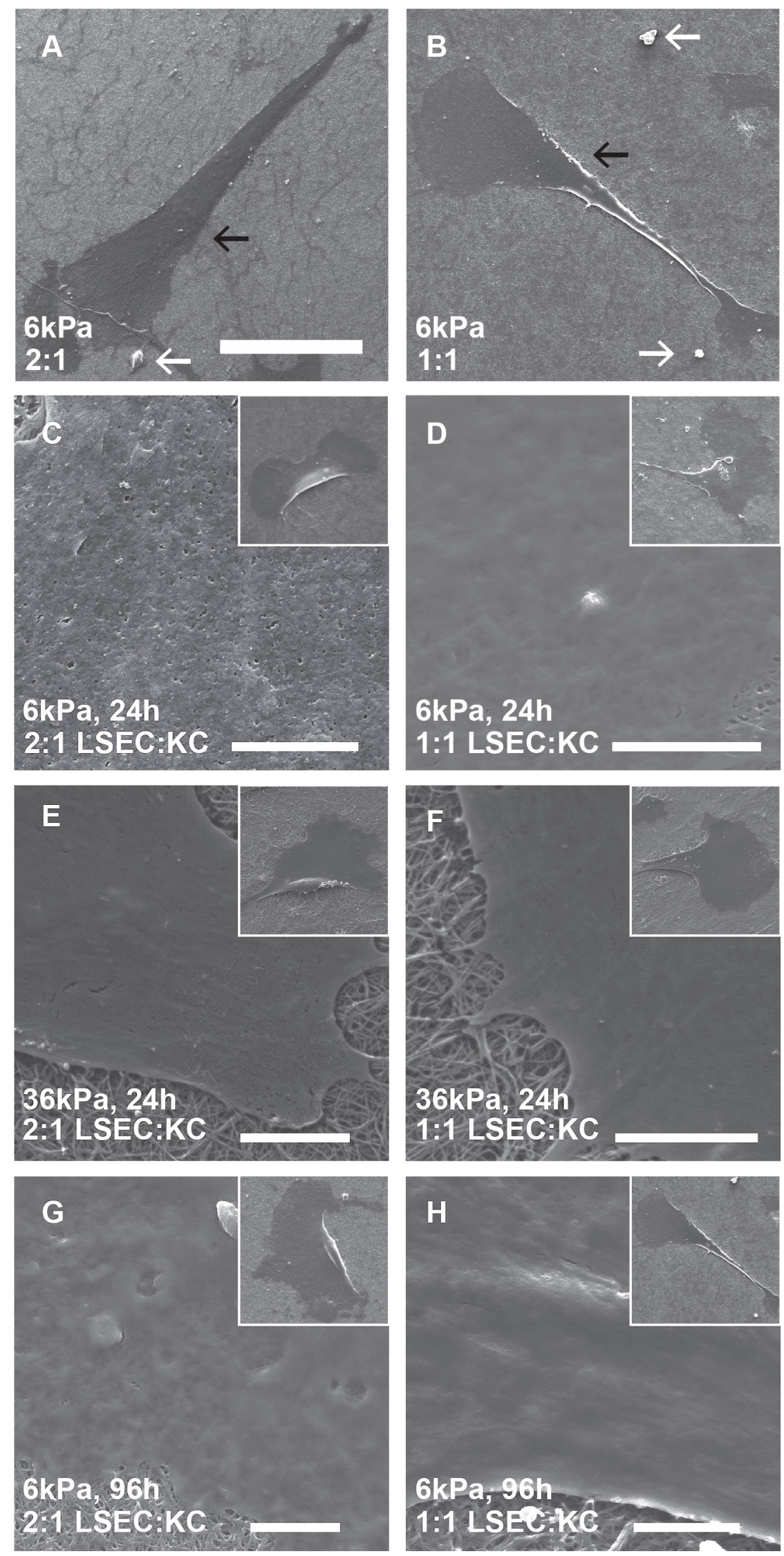

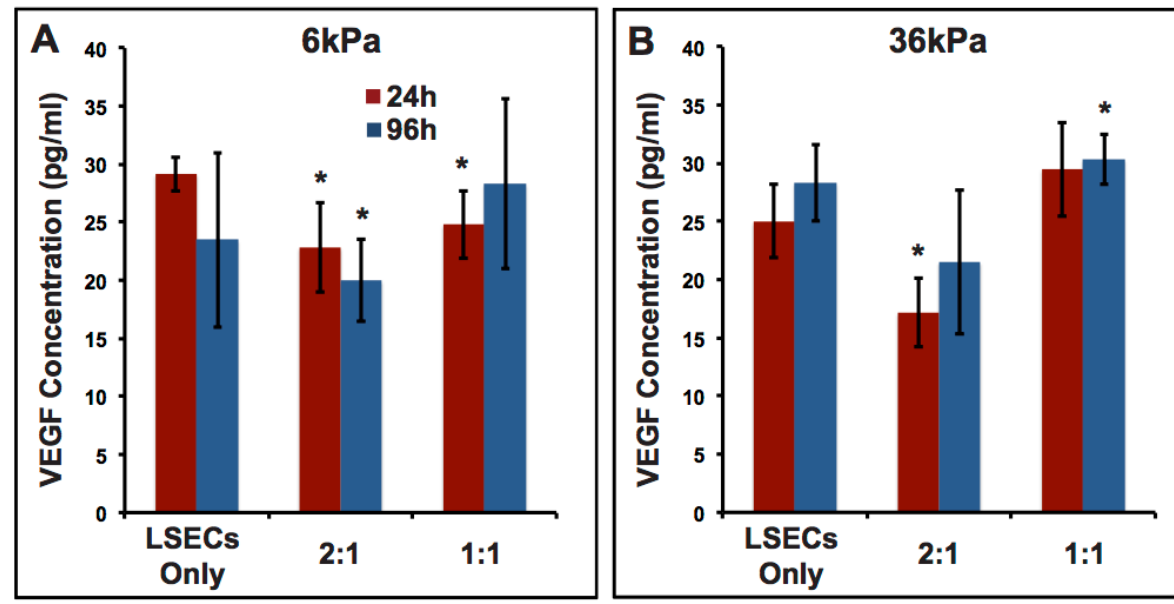

Fig. 3 

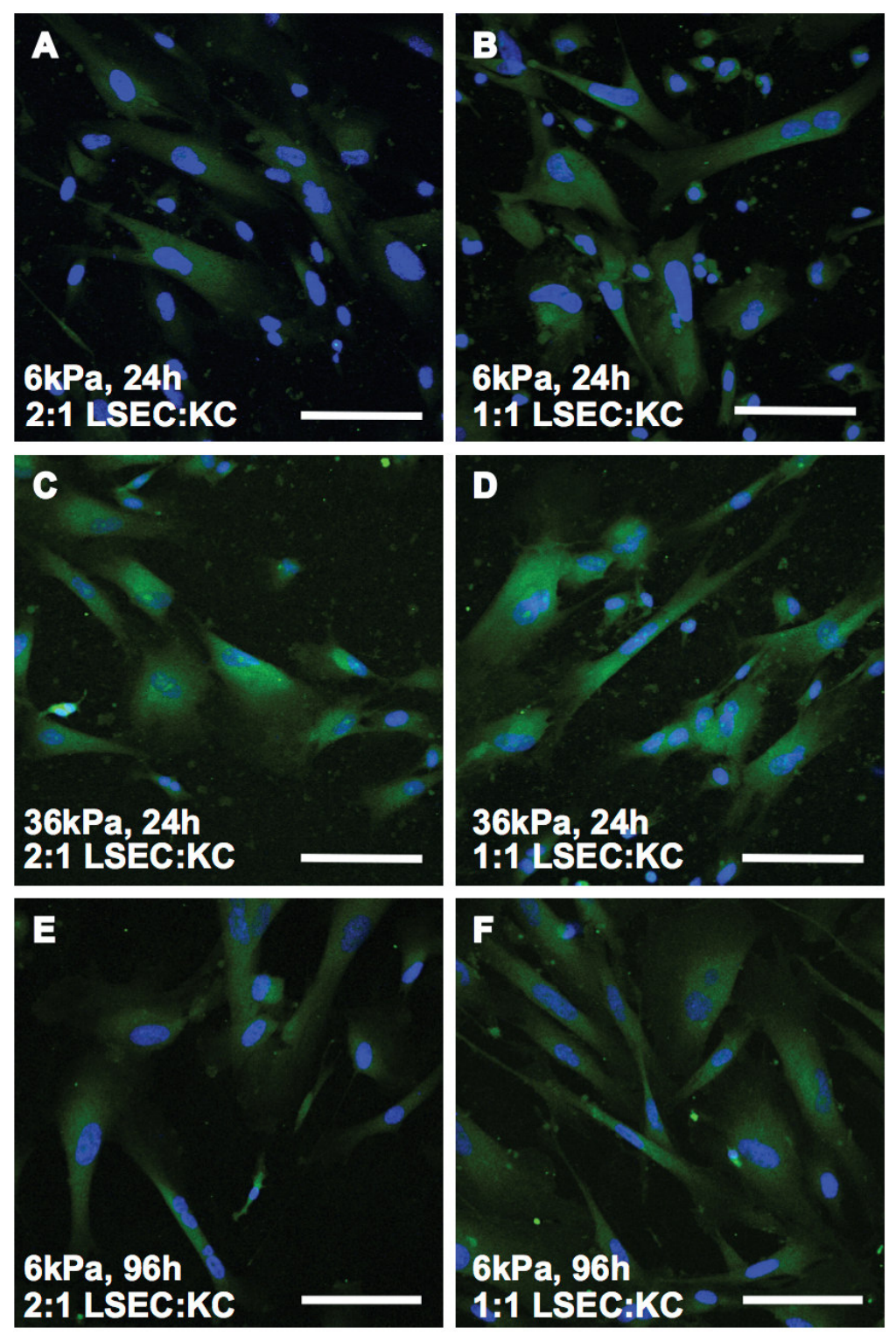

Fig. 4 

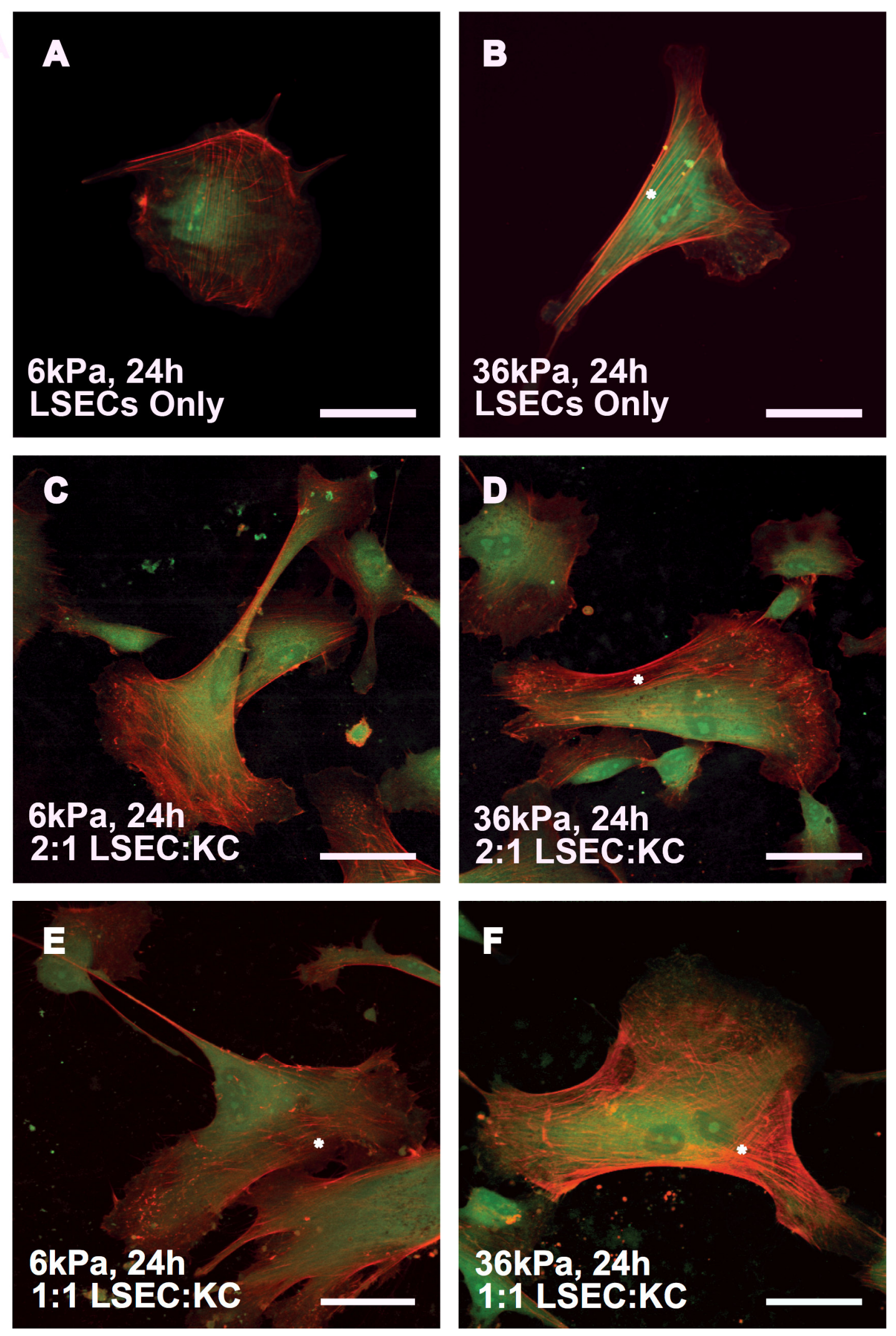

Fig. 5 


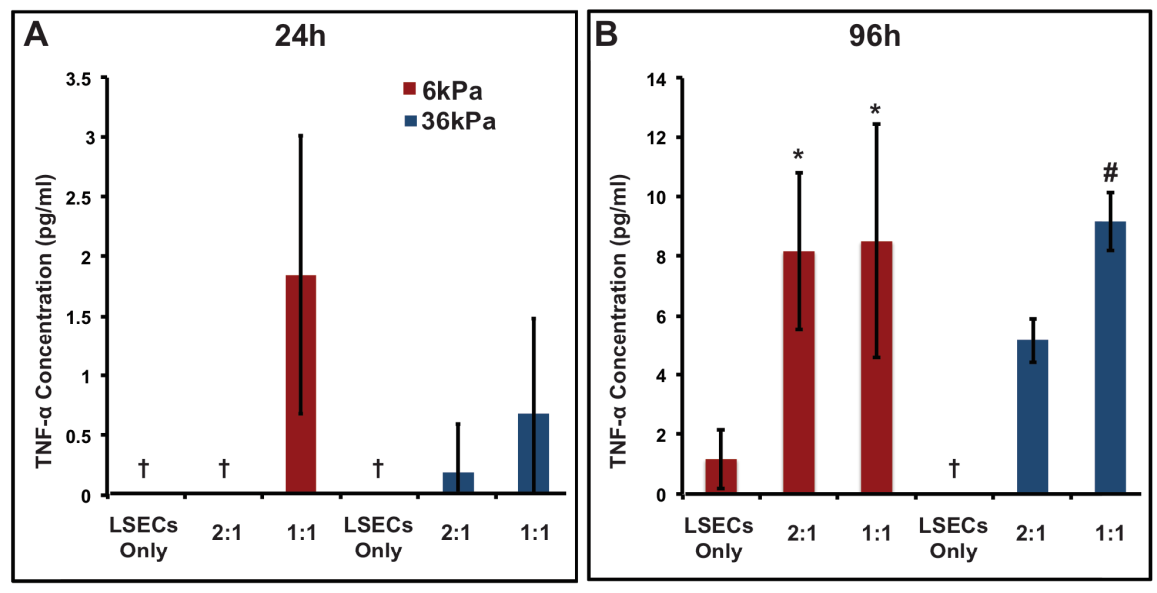

Fig. 6 

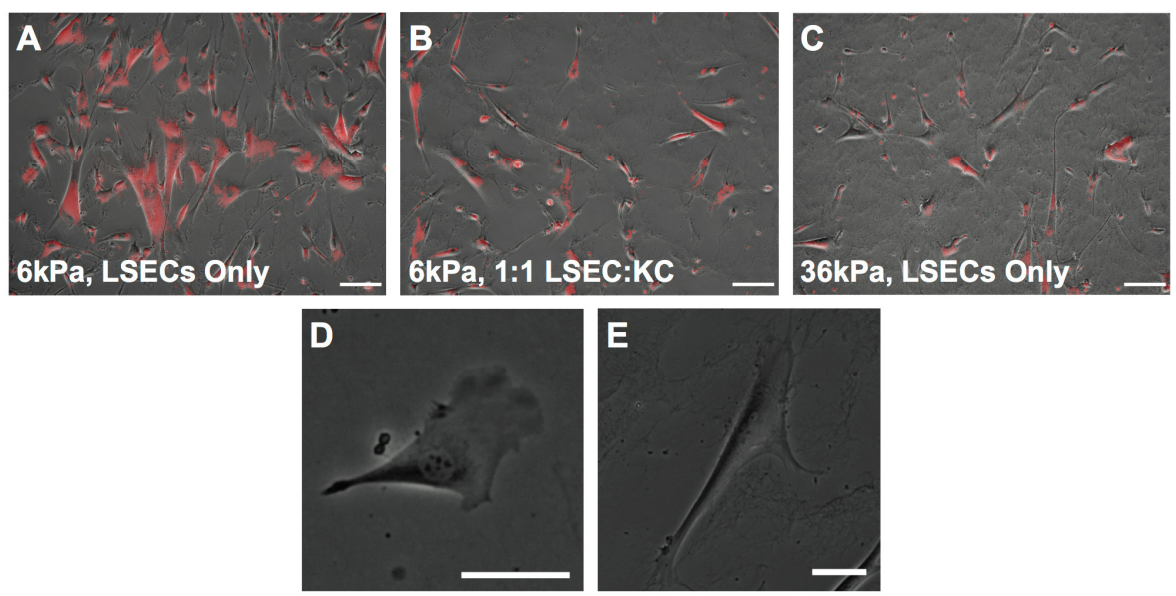

Fig. 7 


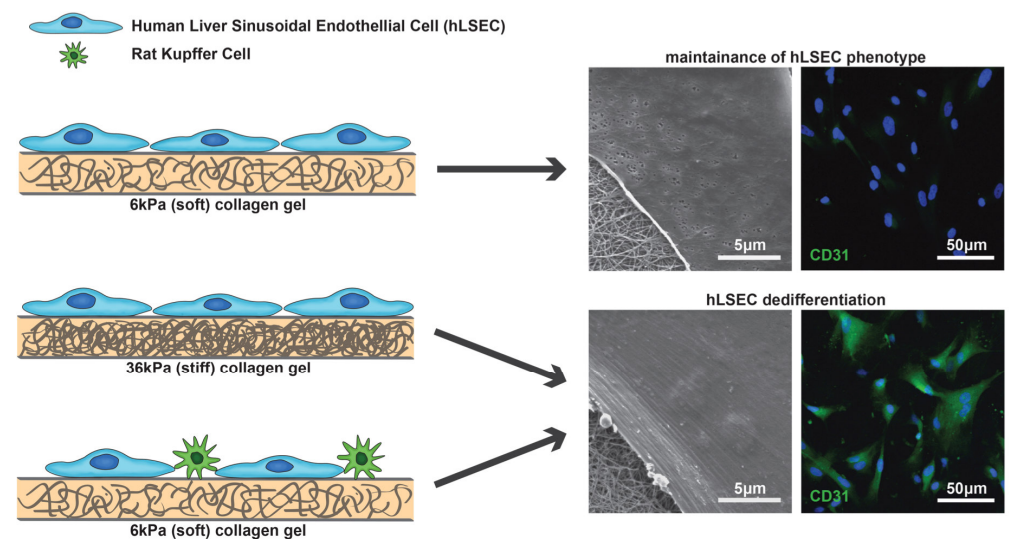

Graphical abstract 